\title{
MBD2 and EZH2 regulate the expression of SFRP1 without affecting its methylation status in a colorectal cancer cell line
}

\author{
JUN YU ${ }^{1,2^{*}}$, YANG XIE ${ }^{1,2^{*}}$, YUTING LIU ${ }^{1,2}$, FENG WANG $^{1,2}$, MENGYING LI $^{1,2}$ and JIAN QI ${ }^{1,2}$ \\ ${ }^{1}$ Department of Gastroenterology, Zhongnan Hospital of Wuhan University; ${ }^{2}$ Hubei Clinical Center and \\ Key Laboratory of Intestinal and Colorectal Diseases, Wuhan, Hubei 430071, P.R. China
}

Received December 1, 2018; Accepted January 22, 2020

DOI: $10.3892 /$ etm.2020.9372

\begin{abstract}
Secreted frizzled-related protein 1 (SFRP1), which is an extracellular inhibitor involved in Wnt signalling, is downregulated by promoter hypermethylation in the early stages of colorectal tumorigenesis. Polycomb (PCG) and methyl-CpG-binding domain (MBD) proteins that serve a role in epigenetic gene regulation. The aim of the present study was to determine the role of PCG and MBD proteins in the regulation of SFRP1 gene expression in colorectal cancer (CRC), specifically in CRC cell lines and the human embryo intestinal mucosa cell line CCC-HIE-2. The methylation status of the SFRP1 gene promoter were analysed using methylation-specific PCR (MSP), whereas SFRP1 mRNA expression was analysed using reverse transcription-quantitative PCR. The association between PCG and MBD proteins and the SFRP1 gene was assessed, where associated proteins were screened by chromatin immunoprecipitation and their expression were subsequently knocked down using RNA interference to determine their role in the regulation of SFRP1 gene expression. The SFRP1 promoter was demonstrated to be hypermethylated in CRC cell lines and partially methylated in the non-cancerous cell line CCC-HIE-2. SFRP1 mRNA expression was significantly lower in CRC cell lines compared with that of CCC-HIE-2 cells. The expression of PCGs enhancer of zeste homolog 2 (EZH2) and BMI1, along with MBD2, was indicated to be upregulated with SFRP1 methylation in HCT116 and SW480 cells. The SFRP1 promoter region was enriched with EZH2 in CCC-HIE-2 cells and enriched with EZH2 and MBD2 in SW480 cells, whereas none of the proteins examined
\end{abstract}

Correspondence to: Dr Jian Qi, Department of Gastroenterology, Zhongnan Hospital of Wuhan University, 169 Donghu Road, Wuhan, Hubei 430071, P.R. China

E-mail: qiqidelizi@163.com

*Contributed equally

Key words: methyl-CpG-binding domain protein 2, enhancer of zeste homolog 2, secreted frizzled related protein 1, colorectal cancer, DNA methylation were indicated on the SFRP1 promoter in HCT116 cells. The expression of SFRP1 was reactivated by MBD2 small interfering (si)RNA but not by EZH2 siRNA in SW480 cells, but combined MBD2 and EZH2 knockdown effectively restored SFRP1 gene expression without affecting the methylation status of the SFRP1 promoter. In conclusion, data from the present study revealed that MBD2 and EZH2 regulated SFRP1 expression without affecting the hypermethylation of SFRP1 in CRC cell lines. Instead, the regulation of SFRP1 expression may be through a distinct mechanism, which warrants further investigation.

\section{Introduction}

Colorectal cancer (CRC) is the third leading cause of cancer-associated mortality in both men and women, and poses a serious health problem (1). Vermeulen et al (2) previously revealed that by modulating Wnt signalling activity, cancer cells can reversibly lose their tumorigenic capabilities, which can be regained following stimulation by myofibroblast-derived factors. This finding demonstrated the plasticity of cancer stem cells, which can be modulated by adjusting the activation status of Wnt signalling (3). The aberrant activation of the Wnt/ $\beta$-catenin signalling pathway is associated with a number of human malignancies, including breast (4), liver (5), lung (6) and colorectal cancer (7).

Secreted frizzled-related proteins (SFRP1-5) belong to a family of soluble proteins that are structurally related to Frizzled proteins. They serve as extracellular inhibitors of Wnt signalling by directly binding to Wnt ligands or Frizzled receptors (8). Downregulated SFRP expression by promoter hypermethylation has been previously demonstrated to result in the constitutive activation of the Wnt pathway, which in turn contributes to CRC malignancy (9). Promoter hypermethylation in the SFRP family of genes have been previously revealed to occur early in the colorectal and adenoma tumorigenesis process (10), where the adenocarcinoma and aberrant crypt foci always maintain a high frequency of SFRP1 methylation, with the rate reaching $94.4 \%$ (11). SFRP gene promoter demethylation has been demonstrated to restore SFRP expression and suppress Wnt signalling, leading to the inhibition of tumour cell proliferation (10). This observation indicated that promoter hypermethylation is associated with the silencing of SFRP gene expression. 
Epigenetic regulation of gene expression does not lead to changes in the actual DNA sequence (12). In eukaryotic cells, gene expression is predominantly regulated by epigenetic modifications the chromatin, which can subsequently adopt active or repressive states (13). For example, histone acetylation, trimethylation and other regulatory factors can render chromatin susceptible to transcriptional activation (13), whilst DNA hypermethylation, chromatin repressors and specific codes of histone methylation can compact the chromatin to repress transcription $(12,13)$. DNA methylation is a particularly important epigenetic modification in the regulation of gene expression. Previous studies have revealed that methylated $\mathrm{CpG}$ islands in the promoter region protrude into the main groove, where transcription factors bind to directly inhibit gene transcription (14).

The polycomb-group (PCG) proteins are a family of epigenetic regulators that can repress transcription through post-translational histone modifications or chromatin compaction (15). Evidence suggests that the overexpression of PCG proteins, and particularly that of BMI1 and EZH2, results in the progression of a variety of cancer types $(16,17)$. The methyl-CpG-binding domain (MBD) protein can specifically recognize and bind to methylated DNA, recruit repressor complexes and histone deacetylases (HDACs) to mediate epigenetic transcriptional repression $(18,19)$. However, to the best of our knowledge, the specific identities of the PCG and MBD proteins that regulate SFRP1 promoter methylation and subsequent SFRP1 expression remain to be determined. Therefore, using CRC cell lines, the aim of the present study was to assess the potential role of PCG and MBD proteins in the regulation of SFRP1 gene expression in CRC.

\section{Materials and methods}

Cell culture. The colon adenocarcinoma cell lines used in the present study were as follows: SW1116 (Dukes A), SW480 (Dukes B) and HCT116 cells (Dukes D), were donated from the Department of Gastroenterology, Zhongnan Hospital of Wuhan University (Wuhan, China) (20). The human embryo intestinal mucosa cell line CCC-HIE-2 (21) (cat. no. 3111C0001CCC000178) was purchased from the China Infrastructure of Cell Line Resources (Beijing, China). All cells were cultured in DMEM (Gibco; Thermo Fisher Scientific, Inc.) supplemented with $10 \%$ (v/v) FBS (Hangzhou Sijiqing Biological Engineering Materials Co., Ltd.) and $1 \%(\mathrm{v} / \mathrm{v})$ penicillin/streptomycin (Thermo Fisher Scientific, Inc.) in a humidified atmosphere at $37^{\circ} \mathrm{C}$ and $5 \% \mathrm{CO}_{2}$.

Methylation-specific PCR (MSP). DNA was extracted from all cell lines using TIANamp Genomic DNA kit (Tiangen Biotech Co., Ltd.) according to manufacturer's protocol. Sodium bisulfite treatment was performed using an EZ DNA Methylation kit (cat. no. D5020; Zymo Research Corp.). The sequences of primers used for the present study were as follows: SFRP1 unmethylated (U) forward, 5'-GTTTTGTAG TTTTTGGAGTTAGTGTTGTGT-3' and reverse, 5'-CCTACG ATCGAAAACGACGCGAACG-3'; SFRP2 (U) forward, 5'-TTTTGGGTTGGAGTTTTTTGGAGTTGTGT-3' and reverse, 5'-AАCCCACTCТСТTCACTAАATACAАCTCA-3'; SFRP4 (U) forward, 5'-GGGGGTGATGTTATTGTTTTT
GTATTGAT-3' and reverse, 5'-CACCTCCCCTAACATAAA CTCAAAACA-3'; SFRP5 (U) forward, 5'-GTAAGATTT GGTGTTGGGTGGGATGTTT-3' and reverse, 5'-AAAACT CCAACCCAAACCTCACCATACA-3'; SFRP1 methylated (M) forward, 5'-TGTAGTTTTCGGAGTTAGTGTCGCGC-3' and reverse, 5'-CCTACGATCGAAAACGACGCGAACG-3', SFRP2 (M) forward, 5'-GGGTCGGAGTTTTTCGGAGTT GCGC-3' and reverse, 5'-CCGCTCTCTTCGCTAAATACG ACTCG-3', SFRP4 (M) forward, 5'-GGGTGATGTTATCGT TTTTGTATCGAC-3' and reverse, 5'-CCTCCCCTAACG TAAACTCGAAACG-3'; SFRP5 (M) forward, 5'-AAGATT TGGCGTTGGGCGGGACGTTC-3' and reverse, 5'-ACT CCAACCCGAACCTCGCCGTACG-3'. The exact locations of the SFRP1, SFRP2, SFRP4 and SFRP5 promoter regions analysed for methylation by MSP are presented in Fig. S1A. Gold Star Taq DNA polymerase (CoWin Biosciences; cat. no. CW0938A) was used to amplify the DNA treated with sodium bisufite. Each $50 \mu$ l reaction mixture consisted of $1 \mu \mathrm{l}$ bisulfite-modified DNA template, $10 \mu \mathrm{l}$ 5X Gold Star Taq PCR Buffer, $4 \mu \mathrm{l}$ dNTP Mix, $2 \mu$ l forward and reverse primer (2.5 mM each), $0.5 \mu \mathrm{l}$ Gold Star Taq DNA Polymerase

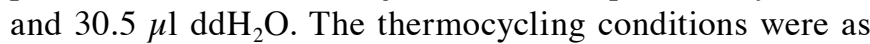
follows: Initial denaturation at $95^{\circ} \mathrm{C}$ for $10 \mathrm{~min}$, followed by 38 cycles of denaturation at $95^{\circ} \mathrm{C}$ for $30 \mathrm{sec}$; annealing at $60^{\circ} \mathrm{C}$ for $30 \mathrm{sec}$, extension at $72^{\circ} \mathrm{C}$ for $30 \mathrm{sec}$ and full extension at $72^{\circ} \mathrm{C}$ for $5 \mathrm{~min}$. The consequent PCR products were then electrophoresed on a $2 \%$ agarose gel.

Chromatin immunoprecipitation (ChIP). ChIP assay was performed using a One-Day Chromatin Immunoprecipitation kit (EMD Millipore) according to the manufacturer's protocol. ChIP was performed using anti-MBD2 (1:50; cat. no. AB38646; Abcam), BMI1 (1:50; cat. no. 5856; Cell Signalling Technology, Inc.) and EZH2 antibodies (1:25; cat. no. 4905; Cell Signalling Technology, Inc.) for $12 \mathrm{~h}$ at $4^{\circ} \mathrm{C}$, with normal rabbit $\mathrm{IgG}$ (cat. no. LK2001; 1:1,000; Sungene Biotech, Ltd.) serving as a control. DNA fragments isolated from chromatin were subjected to 40 cycles of RT-qPCR (cat. no. CW2627; CoWin Biosciences) at $95^{\circ} \mathrm{C}$ for $10 \mathrm{sec}$ followed by annealing at $60^{\circ} \mathrm{C}$ for $60 \mathrm{sec}$ and extension at $95^{\circ} \mathrm{C}$ for $15 \mathrm{sec}$. The enrichment of marks on the target regions was quantified relative to the input amounts. The region of the SFRP1 promoters analysed for methylation by ChIP are presented in Fig. S1B.

RNA interference. All cells $\left(5 \times 10^{5}\right.$ cells/well) were seeded into six-well plates in DMEM, then $100 \mathrm{nM}$ MBD2, EZH2 or a negative control small interfering (si)RNA was transfected into cells using Lipofectamine JET (Invitrogen; Thermo Fisher Scientific, Inc; cat. no. SL100468) according to the manufacturer's protocols. After $24 \mathrm{~h}$ incubation at $37^{\circ} \mathrm{C}$ in a $\mathrm{CO}_{2}$ incubator, the medium was replaced with fresh DMEM containing 10\% FBS and siRNA-Lipofectamine JET complexes $24 \mathrm{~h}$ prior to further culture. The sequences of siRNA used for the present study were as follows: MBD2-001 forward, 5'-GAGGCUACAAGGACUUAGUTT-3' and reverse, 5'-ACUAAGUCCUUGUAGCCUCTT-3'. MBD2-002, 5'-GAU GAUGCCUAGUAAAUUATT-3' and reverse, 5'-UAAUUU ACUAGGCAUCAUCTT-3'. MBD2-003, 5'-CCUGGGAAA UACUGUUGAUTT-3' and reverse, 5'-AUCAACAGUAUU UCCCAGGTT-3'. EZH2-2196 forward, 5'-GCAGCUUUC 
UGUUCAACUUTT-3' and reverse, 5'-AAGUUGAACAGA AAGCUGCTT-3'. EZH2-488 forward, 5'-GACUCUGAA UGCAGUUGCUTT-3' and reverse, 5'-AGCAACUGCAUU CAGAGUCTT-3'. EZH2-1952 forward, 5'-CCUGACCUC UGUCUUACUUTT-3' and reverse, 5'-AAGUAAGACAGA GGUCAGGTT-3'. A nonspecific control siRNA was used as a control. NC forward, 5'-UUCUCCGAACGUGUCACGUTT-3' and reverse, 5'-ACGUGACACGUUCGGAGAATT-3'.

cDNA preparation and reverse transcription-quantitative $P C R(R T-q P C R)$. Total RNA was isolated from cells using an easy- spin $^{\mathrm{TM}}$ Total RNA extraction kit (Beijing, Aidlab Biotechnologies Co., Ltd.) according to the manufacturer's protocols. The concentration of RNA was measured using a NanoDrop ${ }^{\mathrm{TM}} 2000$ spectrophotometer (Thermo Fisher Scientific, Inc.). A total of 1,000 ng total RNA was used for cDNA synthesis using a ReverTra Ace ${ }^{\circledR}$ qPCR RT kit (Toyobo Life Science) and a temperature protocol of $15 \mathrm{~min}$ at $37^{\circ} \mathrm{C}$, $5 \mathrm{~min}$ at $98^{\circ} \mathrm{C}$ and $4^{\circ} \mathrm{C}$ thereafter. RT-qPCR was subsequently performed using SYBR ${ }^{\circledR}$ Green PCR Master Mix (cat. no. CW2627; ComWin Biosciences) in a Bio-Rad 7500 Real-time PCR System (Bio-Rad Laboratories, Inc.). Primers used for qPCR contained the following sequences: GAPDH forward, 5'-AGAAGGCTGGGGCTCATTTG-3' and reverse, 5'-GCAGGAGGCATTGCTGATGAT-3'; EZH2 forward, 5'-TCCTACATCCTTTTCATGCAACAC-3' and reverse, 5'-GCTCCCTCCAAATGCTGGTA-3'; MBD2 forward, 5'-GCAAGCCTCAGTTGGCAAG-3' and reverse, 5'-ATC GTTTCGCAGTCTCTGTTT-3'; SFRP1 forward, 5'-TGG CCCGAGATGCTTAAGTG-3', and reverse, 5'-GACACACCG TTGTGCCTTG-3'; SFRP2 forward, 5'-CGACATGCTTGA GTGCGAC-3' and reverse, 5'-CTTTGGAGTTCCTCGGTG G-3'; SFRP4 forward, 5'-TCACCCATCCCTCGAACTCA-3' and reverse, 5'-CATCATCCTTGAGCGCCACT-3'; SFRP5 forward, 5'-CCTCCAGTGACCAAGATCTGC-3' and reverse, 5'-TCCTTGATGCGCATTTTGACC-3'. GAPDH was used as an internal reference. The thermocycling conditions were as follows: Initial denaturation at $95^{\circ} \mathrm{C}$ for $10 \mathrm{~min}$, followed by 39 cycles of denaturation at $95^{\circ} \mathrm{C}$ for $10 \mathrm{sec}$, annealing at $60^{\circ} \mathrm{C}$ for $20 \mathrm{sec}$, extension at $72^{\circ} \mathrm{C}$ for $10 \mathrm{sec}$ and final extension at $72^{\circ} \mathrm{C}$ for $3 \mathrm{~min}$. All reactions were performed in triplicate, and the results were analysed and expressed relative to threshold cycle $(\mathrm{Cq})$ values and then converted to fold change values $\left(2^{-\Delta \Delta C q}\right)(22)$.

Statistical analysis. SPSS 20.0 software (IBM Corp.) was used for data analysis. Data presented were analysed using one-way ANOVA followed by a Bonferroni's correction and presented as mean \pm standard deviation in the text and figures. $\mathrm{P}<0.05$ was considered to indicate a statistically significant difference.

\section{Results}

DNA promoter hypermethylation and reduced SFRPI $m R N A$ expression in CRC cell lines. The SFRP1 gene promoter was demonstrated to be completely methylated in SW1116, SW480 and HCT116 cells but only partially methylated in CCC-HIE-2 cells according to MSP analysis (Fig. 1). The mRNA levels of SFRP1 was significantly lower in CRC cell lines compared with that of CCC-HIE-2 (Fig. 2).

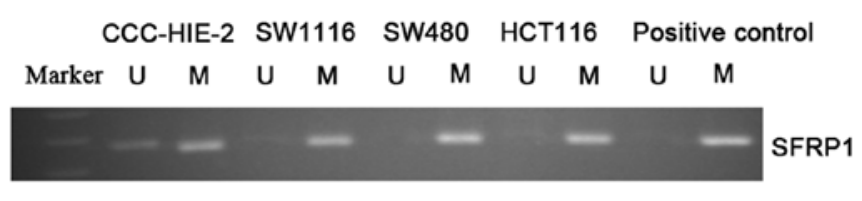

Figure 1. MSP of SFRP1 in CRC cell lines SW1116, SW480 and HCT116 cells and a normal intestinal mucosal cell line CCC-HIE-2 cells. SFRP1, Secreted frizzled-related protein 1; M, methylated DNA product amplified using methylation-specific primers; $\mathrm{U}$, unmethylated DNA product amplified using nonmethylation-specific primers; MSP, Methylation-specific PCR; Positive control, Placental DNA modified by $\mathrm{CpG}$ methylase; CRC, colorectal cancer.

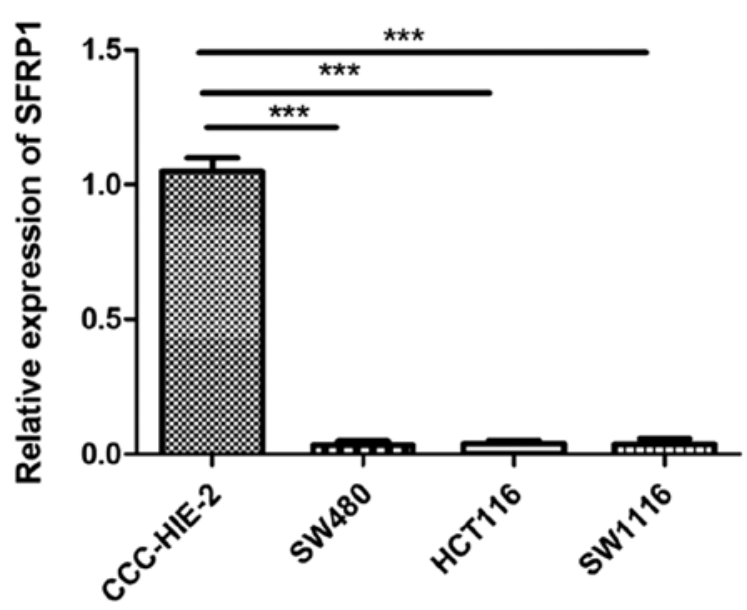

Figure 2. SFRP1 mRNA expression in CRC cell lines SW1116, SW480 and HCT116 cells and a normal intestinal mucosal cell line CCC-HIE-2 cells, measured using reverse transcription-quantitative PCR. GAPDH was used as an internal quantitative control and CCC-HIE-2 as external controls. Three independent experiments were performed. ${ }^{* * *} \mathrm{P}<0.001$. SFRP1, secreted frizzled-related protein 1 ; CRC, colorectal cancer.

Expression of EZH2, BMI1 and MBD2 are upregulated with SFRP1 methylation in HCT116 and SW480 cells. EZH2 mRNA expression was revealed to be significantly increased in CRC cell lines compared with CCC-HIE-2 cells. MBD2 and BMI1 expression were significantly higher in SW480 and HCT116 cells compared with CCC-HIE-2 cells, but was markedly lower in SW1116 cells compared with CCC-HIE-2 cells (Fig. 3).

EZH2 and MBD2 can bind to the promoter region of SFRP1. To examine if PCG and MBD proteins can interact with the SFRP1 promoter directly, ChIP assay was performed in HCT116, SW480 and CCC-HIE-2 cell samples. Enrichment of EZH2 (\% input $=2.899$ ) on the SFRP1 promoter region was observed in CCC-HIE-2 cell samples. In SW480 cells, increased binding of MBD2 (\% input $=3.244)$ and reduced binding of EZH2 (\% input $=1.147$ ) to the SFRP1 promoter were observed compared with samples from CCC-HIE-2 cells. However, no enrichment was observed in samples from HCT116 cells (Fig. 4).

Knockdown of MBD2 and EZH2 expression increases SFRP1 expression in SW480 cells. To investigate the effect of MBD2 and EZH2 on SFRP1 gene expression and promoter methylation, MBD2 and EZH2 knockdown experiments were performed on SW480 cells following confirmation 

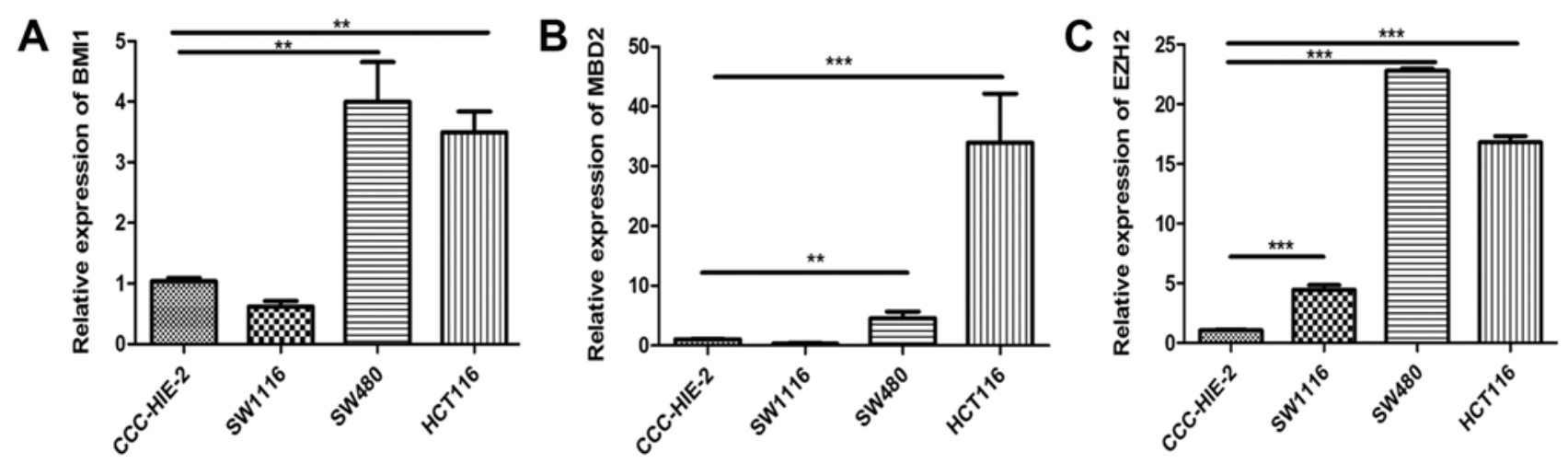

Figure 3. BMI1, MBD2 and EZH2 mRNA expression in CRC cell lines SW1116, SW480 and HCT116 cells and a normal intestinal mucosal cell line CCC-HIE-2 cells, measured using reverse transcription-quantitative PCR. GAPDH was used as an internal quantitative control and CCC-HIE-2 as external controls. (A) BMI1 mRNA expression. (B) MBD2 mRNA expression. (C) EZH2 mRNA expression. Three independent experiments were performed. ${ }^{* *} \mathrm{P}<0.01$ and ${ }^{* * *} \mathrm{P}<0.001$. MBD2, methyl-CpG-binding domain protein 2; EZH2, enhancer of zeste homolog 2.

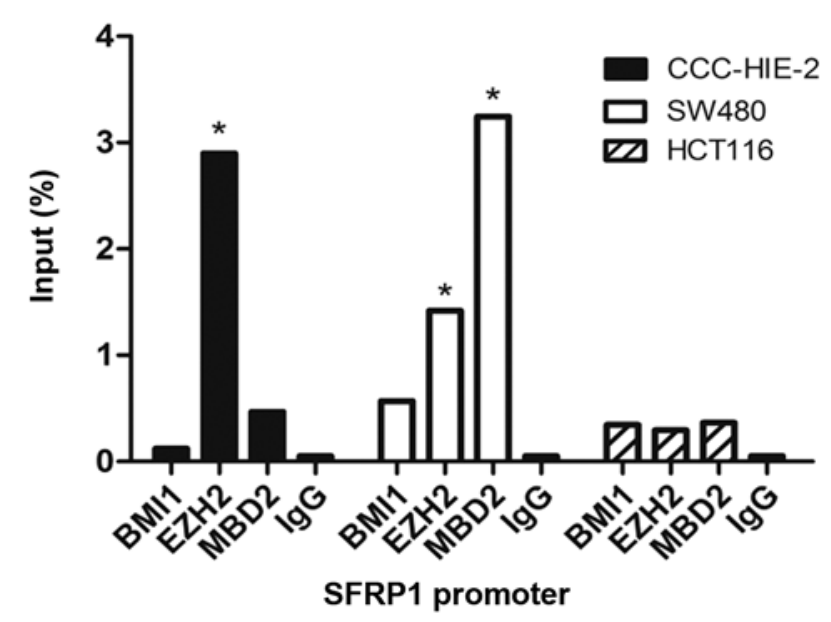

Figure 4. Enrichment of MBD2, EZH2 and BMI1 on the SFRP1 promoter in CCC-HIE-2, SW480 and HCT116 cells on DNA isolated using chromatin immunoprecipitation. ${ }^{*} \mathrm{P}<0.05$. MBD2, methyl-CpG-binding domain protein 2; EZH2, enhancer of zeste homolog 2; SFRP1, secreted frizzled-related protein 1.

of siRNA transfection efficiency (Fig. 5A and B). SFRP1 expression was demonstrated to be significantly higher following transfection with MBD2 siRNA but not EZH2 siRNA (Fig. 5C). However, co-transfection of SW480 cells with EZH2 siRNA alongside MBD2 siRNA significantly potentiated the increase in SFRP1 gene expression compared with MBD2 siRNA alone (Fig. 5C). The methylation status of the SFRP promoters was therefore indicated to not be altered by MBD2 or EZH2 knockdown, either alone or in combination (Fig. 6A-C).

\section{Discussion}

SFRP proteins have been demonstrated to directly and indirectly inhibit Wnt signalling (23). Accumulating evidence has suggested that SFRP expression is tightly regulated by promoter methylation (24). In the present study, SFRP1 mRNA expression was significantly downregulated in CRC cell lines compared with CCC-HIE-2 cells, a non-cancerous intestinal mucosal cell line, with concomitant hypermethylation in the SFRP1 promoter region. Therefore, it was hypothesized that the silencing of SFRP1 expression was due to methylation of the SFRP1 gene promoter.

DNA methyltransferase (DNMT), which catalyses the transfer of methyl groups to the carbon-5 position of cytosine in the dinucleotide sequence $\mathrm{CpG}$, is associated with establishing and maintaining the profile of DNA methylation (25). A previous study has demonstrated that treatment of SW480 and HCT116 cells with the DNA methylation inhibitor decitabine (DAC) and HDAC inhibitor trichostatin A (TSA) resulted in the restoration of SFRP1 expression, which was markedly higher compared with that observed with DAC treatment only; though TSA treatment alone did not restore the expression of SFRP1 (11). DAC is a DNMT inhibitor that specifically promotes the degradation of DNMT1, whereas TSA is a histone deacetylase inhibitor resulting in hyperacetylation on histones $\mathrm{H} 3$ and $\mathrm{H} 4$ (26). This result suggests that promoter hypermethylation of SFRP1 was partly mediated by DNMT1 and downregulation of SFRP1 in SW480 and HCT116 cells was mediated by both DNA methylation and histone deacetylation.

A previous study reported that BMI1 interacts directly with DNMT1-associated protein 1, which in turn interacts with DNMT1 to mediate transcriptional repression (27). EZH2 can recruit DNMT1, DNMT3A and DNMT3B to promote DNA methylation at $\mathrm{CpG}$ islands and establish stable repressive chromatin structures (19). MBD2, which is a member of the MBD protein family, can specifically recognize and bind to methylated $\mathrm{CpG}$ sites, recruit co-repressors and modify the chromatin structure to inhibit gene transcription, with HDAC as an example (28). MBD2 provides a potential link between DNA methylation, chromatin remodelling and histone modification. Enhanced binding of DNMT1, HDAC1 and MBD proteins to the transcriptional start site of SFRP1 in hepatocarcinoma cells has been previously reported to result in the epigenetic silencing of SFRP1 expression (29).

According to results in the present study, the transcription levels of MBD2 and BMI1 were only observed to be upregulated in SW480 and HCT116 cells, whilst EZH2 expression was increased from the early stages of CRC onwards, suggesting that EZH2 may be involved in the occurrence of early CRC. The involvement of BMI1, MBD2 and EZH2 in the regulation of DNMT and HDAC migration mainly occurred during the middle to late stages of CRC development. 
A

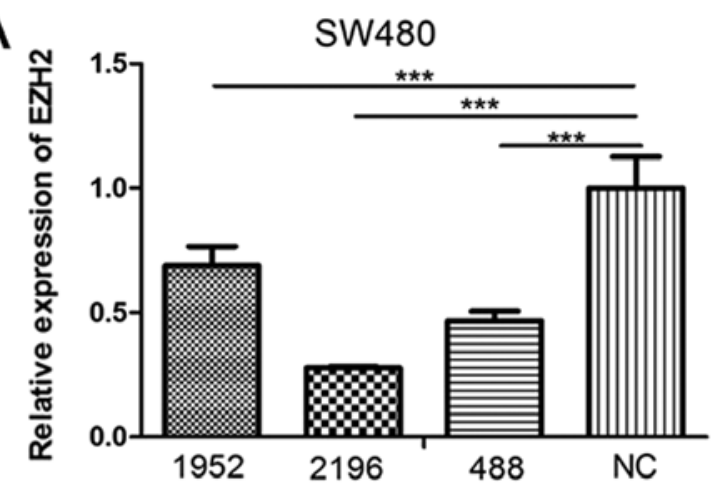

B

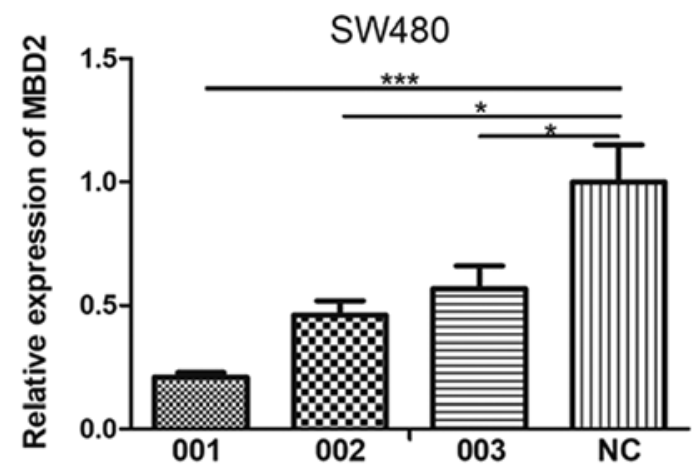

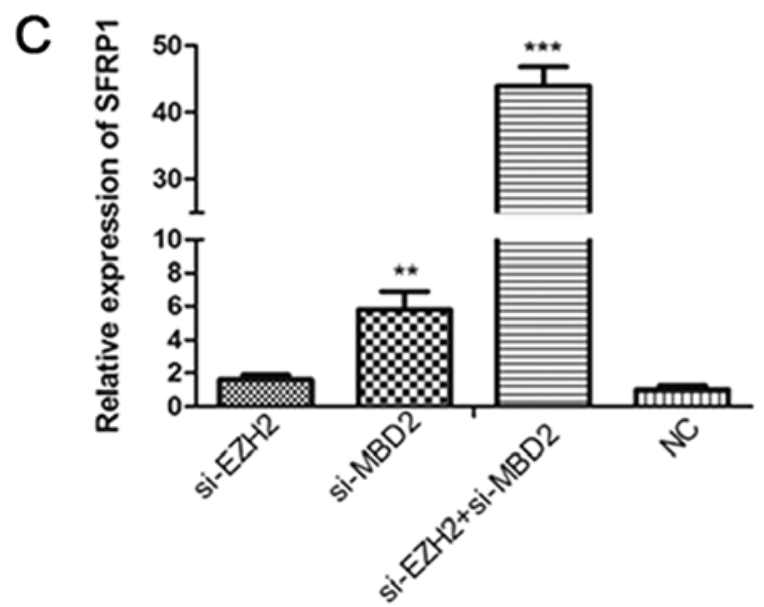

Figure 5. Effects of MBD2 and EZH2 knockdown on the expression of SFRP1. (A) Reverse transcription-quantitative PCR analysis of EZH2 mRNA expression in SW480 cells transfected with EZH2 or NC siRNA. (B) Reverse transcription-quantitative PCR analysis of MBD2 mRNA expression in SW480 cells transfected with MBD2 or NC siRNA. (C) Expression of SFRP1 in SW480 cells was detected by reverse transcription-quantitative PCR following MBD2 and/or EZH2 knockdown. GAPDH was used as an internal control. ${ }^{*} \mathrm{P}<0.05,{ }^{* *} \mathrm{P}<0.01,{ }^{* * *} \mathrm{P}<0.001$. si, small interfering RNA; NC, negative control; MBD2, methyl-CpG-binding domain protein 2; EZH2, enhancer of zeste homolog 2; SFRP1, secreted frizzled-related protein 1.

A

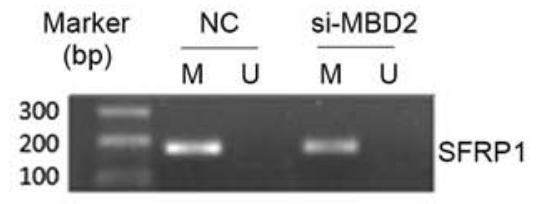

B

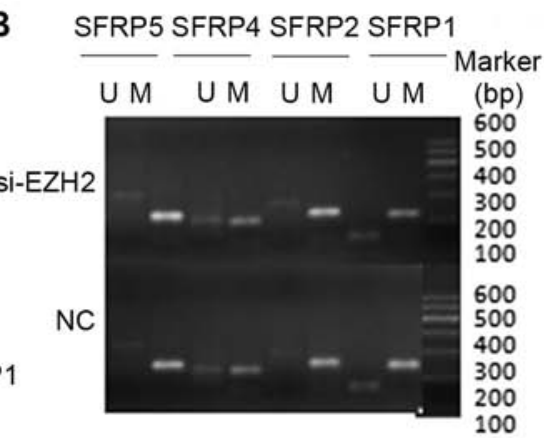

C

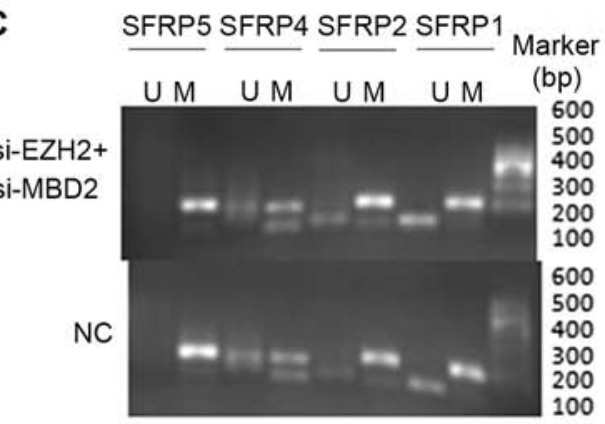

Figure 6. Effect of MBD2 and/or EZH2 knockdown on the methylation status of SFRP1 in SW480 cells. (A) MSP of the SFRP1 promoter after knocking down MBD2, (B) EZH2 and (C) MBD2 and EZH2 expression. si, small interfering RNA; NC, negative control; MBD2, methyl-CpG-binding domain 2; EZH2, enhancer of zeste homolog 2; SFRP1, secreted frizzled-related protein 1; M, methylated DNA product amplified using methylation-specific primers; $\mathrm{U}$, unmethylated DNA product amplified using nonmethylation-specific primers; MSP, methylation-specific PCR.

To clarify the mechanism involved in the silencing of SFRP1 by PCG and MBD proteins, potential PCG-SFRP1 promoter and MBD-SFRP1 promoter complexes were detected using ChIP in CCC-HIE-2, SW480 and HCT116 cells. BMI1 did not combine with the SFRP1 promoter region in the three cell lines, suggesting that it did not participate in SFRP1 promoter methylation. The enrichment of EZH2 on the SFRP1 promoter region of SW480 cells was considerably lower compared with that in CCC-HIE-2 cells, suggesting that it may be associated with SFRP1 promoter methylation in CCC-HIE-2 cells but not in SW480 cells. MBD2 was only indicated on the SFRP1 promoter in SW480 cells, indicative of a role in SFRP1 promoter methylation during the middle stages of CRC. In addition, it was revealed that MBD2 and EZH2 bound to the SFRP1 gene promoter in SW480 cells but not in HCT116 cells, suggesting that these two proteins may serve distinct regulatory functions during CRC tumorigenesis. In SW480 cells, SFRP1 gene expression was restored following MBD2 knockdown but not after EZH2 knockdown. In contrast, when MBD2 


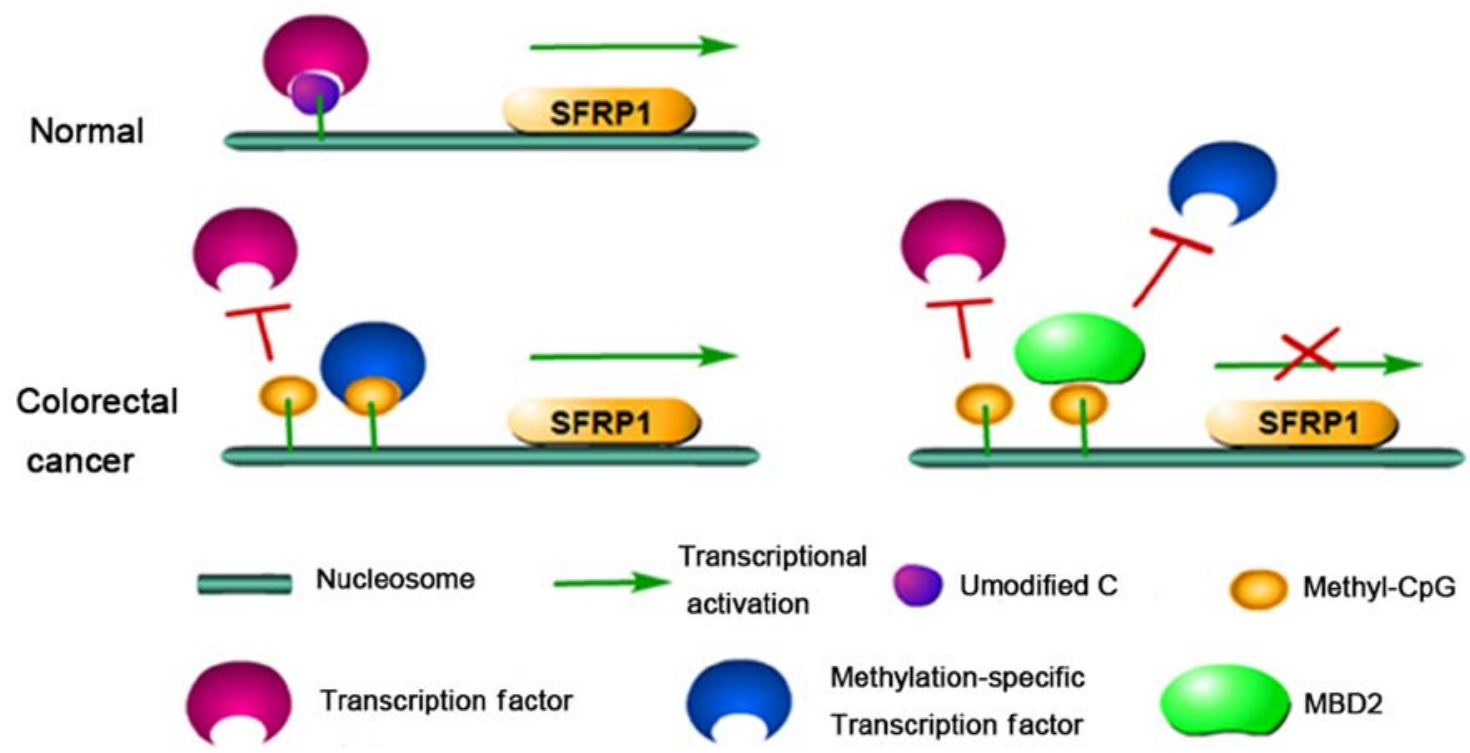

Figure 7. Silencing model of the SFRP1 gene in colorectal cancer cells. SFRP1, secreted frizzled-related protein 1; MBD2, methyl-CpG-binding domain protein 2.

and EZH2 were knocked down simultaneously, the expression of SFRP1 was found to be significantly increased. However, neither MBD2 or EZH2 knockdown could reverse SFRP1 gene promoter hypermethylation. These observations suggest that SFRP1 gene inactivation in CRC is associated with an additional mechanism that is distinct from promoter hypermethylation.

MBD2 has been previously revealed to bind to methylated DNA in the promoter region and recruit the Mi-2/nucleosome remodelling and deacetylase nucleosome remodelling complex, resulting in histone deacetylation and chromatin remodelling followed by inhibition of gene transcription $(18,30)$. This effect can be potentiated by EZH2, which also functions in chromatin remodelling $(18,30)$.

Inhibition of MBD2 and EZH2 can reduce the activity of histone deacetylase and alleviate the repressive state of chromatin, restoring gene expression (31). This phenomenon is an indirect mechanism of inhibiting gene expression without changing DNA methylation status. However, a previous study demonstrated that histone deacetylation was not associated with the suppression of SFRP1 gene expression, as inhibition of histone deacetylase alone did not restore SFRP1 expression (11). This mechanism is not sufficient to explain the role of MBD2 knockdown in the restoration of expression. A possible reason could be that SFRP1 transcription persisted even with DNA methylation.

Studies have indicated that certain transcription factors can also bind methylated DNA $(32,33)$. CG dinucleotides in the promoter region of SFRP1 can associate with different types of transcription factors depending on the methylation status of the sequence. In CRC cells, the hypermethylated promoter of SFRP1 blocks transcription factor binding to unmethylated CG, which may explain why the hypermethylation of the SFRP1 promoter in colorectal tumours effectively restores gene expression, whilst the other transcription factors that preferentially bind to methylated CG of SFRP1 can still maintain gene transcription (34). Therefore, it can be speculated that MBD2 may recognize and bind to the methylated promoter of SFRP1, outcompeting other transcription factors that would normally bind to the methylated $\mathrm{CG}$, resulting in the complete inactivation of the SFRP1 gene in CR tumours (Fig. 7). This could explain the restoration of SFRP1 gene expression without changes in the methylation status of the SFRP1 promoter following EZH2 and MBD2 knockdown.

The present study demonstrated that MBD2 and EZH2 regulated SFRP1 expression without affecting SFRP1 methylation in CRC cell lines. Further studies should be undertaken by profiling transcription factors that can bind to the SFRP1 gene promoters. In particular, the role of the typical transcription factors on the restoration of SFRP1 expression, the precise mechanistic function of MBD2 and EZH2 in addition to the regulatory mechanism of DNA methylation on SFRP1 expression in CR tumours require further study.

\section{Acknowledgements}

The abstract was presented at the 26th United European Gastroenterology (UEG) Week, October 20-24, 2018 in Vienna, Austria.

\section{Funding}

This project was supported by the Chinese National Science Foundation Projects (grant no. 81101868) and the Applied Basic Research Programs of Wuhan Science and Technology Department (grant no. 2015061701011642).

\section{Authors' contributions}

All authors were responsible for the conception and design of the present study. JY, YX and YL were responsible for the provision of the study materials. JY and YX were responsible for the collection and assembly of the data. JY, YX, YL, FW, ML and JQ performed the data analysis and interpretation. JY, YX and JQ contributed in writing the manuscript. JY, YX, YL, FW, ML and JQ read and gave the final approval of the manuscript. All authors read and approved the final manuscript. 


\section{Availability of data and materials}

The datasets used and/or analyzed during the current study are available from the corresponding author on reasonable request.

\section{Ethics approval and consent to participate}

Not applicable.

\section{Patient consent for publication}

Not applicable.

\section{Competing interests}

The authors declare that they have no competing interests.

\section{References}

1. Siegel R, Desantis C and Jemal A: Colorectal cancer statistics, 2014. CA Cancer J Clin 64: 104-117, 2014.

2. Vermeulen L, De Sousa E Melo F, van der Heijden M, Cameron K, de Jong JH, Borovski T, Tuynman JB, Todaro M, Merz C, Rodermond $\mathrm{H}$, et al: Wnt activity defines colon cancer stem cells and is regulated by the microenvironment. Nat Cell Biol 12: 468-476, 2010.

3. Jensen M, Hoerndli FJ, Brockie PJ, Wang R, Johnson E, Maxfield D, Francis MM, Madsen DM and Maricq AV: Wnt signaling regulates acetylcholine receptor translocation and synaptic plasticity in the adult nervous system. Cell 149: 173-187, 2012.

4. Lv C, Li F, Li X, Tian Y, Zhang Y, Sheng X, Song Y, Meng Q, Yuan S, Luan L, et al: MiR-31 promotes mammary stem cell expansion and breast tumorigenesis by suppressing Wnt signaling antagonists. Nat Commun 8: 1036, 2017.

5. Russell JO and Monga SS: Wnt/ $\beta$-catenin signaling in liver development, homeostasis, and pathobiology. Annu Rev Pathol 13: 351-378, 2018

6. Tammela T, Sanchez-Rivera FJ, Cetinbas NM, Wu K, Joshi NS, Helenius K, Park Y, Azimi R, Kerper NR, Wesselhoeft RA, et al: A Wnt-producing niche drives proliferative potential and progression in lung adenocarcinoma. Nature 545: 355-359, 2017.

7. Klaus $\mathrm{A}$ and Birchmeier W: Wnt signalling and its impact on development and cancer. Nat Rev Cancer 8: 387-398, 2008.

8. Rattner A, Hsieh JC, Smallwood PM, Gilbert DJ, Copeland NG, Jenkins NA and Nathans J: A family of secreted proteins contains homology to the cysteine-rich ligand-binding domain of frizzled receptors. Natl Acad Sci USA 94: 2859-2863, 1997.

9. Suzuki H, Watkins DN, Jair KW, Schuebel KE, Markowitz SD Chen WD, Pretlow TP, Yang B, Akiyama Y, Van Engeland M, et al: Epigenetic inactivation of SFRP genes allows constitutive WNT signaling in colorectal cancer. Nat Genet 36: 417-422, 2004.

10. Elzi DJ, Song MH, Hakala K, Weintraub ST and Shiio Y: Wnt antagonist SFRP1 functions as a secreted mediator of senescence. Mol Cell Biol 32: 4388-4399, 2012.

11. Qi J, Zhu YQ, Luo J and Tao WH: Hypermethylation and expression regulation of secreted frizzled-related protein genes in colorectal tumor. World J Gastroenterol 12: 7113-7117, 2006.

12. Margueron R and Reinberg D: Chromatin structure and the inheritance of epigenetic information. Nat Rev Genet 11: 285-296, 2010.

13. Allis CD and Jenuwein T: The molecular hallmarks of epigenetic control. Nat Rev Genet 17: 487-500, 2016.

14. Hughes TR and Lambert SA: Transcription factors read epigenetics. Science 356: 489-490, 2017.
15. Riising EM, Comet I, Leblanc B, Wu X, Johansen JV and Helin K: Gene silencing triggers polycomb repressive complex 2 recruitment to CpG islands genome wide. Mol Cell 55: 347-360, 2014.

16. Pan H, Bilinovich SM, Kaur P, Riehn R, Wang $\mathrm{H}$ and Williams DC Jr: CpG and methylation-dependent DNA binding and dynamics of the methylcytosine binding domain 2 protein at the single-molecule level. Nucleic Acids Res 45: 9164-9177, 2017.

17. Negishi M, Saraya A, Miyagi S, Nagao K, Inagaki Y,Nishikawa M, Tajima S, Koseki H, Tsuda H, Takasaki Y, et al: Bmil cooperates with Dnmt1-associated protein 1 in gene silencing. Biochem Biophys Res Commun 353: 992-998, 2007.

18. Wood KH and Zhou Z: Emerging molecular and biological functions of MBD2, a reader of DNA methylation. Fron Genet 7: 93 , 2016.

19. Vire E, Brenner C, Deplus R, Blanchon L, Fraga M, Didelot C, Morey L, Van Eynde A, Bernard D, Vanderwinden JM, et al: The Polycomb group protein EZH2 directly controls DNA methylation. Nature 439: 871-874, 2006.

20. Ahmed D, Eide PW, Eilertsen IA, Danielsen SA, Eknæs M, Hektoen M, Lind GE and Lothe RA: Epigenetic and genetic features of 24 colon cancer cell lines. Oncogenesis 2: e71, 2013.

21. Bai J, Xu J, Zhao J and Zhang R: IncRNA SNHG1 cooperated with miR-497/miR-195-5p to modify epithelial-mesenchymal transition underlying colorectal cancer exacerbation. J Cell Physiol 235: 1453-1468, 2020.

22. Livak KJ and Schmittgen TD: Analysis of relative gene expression data using real-time quantitative PCR and the 2(-Delta Delta C(T)) method. Methods 25: 402-408, 2001

23. Cruciat CM and Niehrs C: Secreted and transmembrane wnt inhibitors and activators. Cold Spring Harb Perspect Biol 5: a015081, 2013.

24. Yu J, Xie Y, Li M, Zhou F, Zhong Z, Liu Y, Wang F and Qi J: Association between SFRP promoter hypermethylation and different types of cancer: A systematic review and meta-analysis. Oncol Lett 18: 3481-3492, 2019.

25. Tatematsu KI, Yamazaki T and Ishikawa F: MBD2-MBD3 complex binds to hemi-methylated DNA and forms a complex containing DNMT1 at the replication foci in late $\mathrm{S}$ phase. Genes Cells 5: 677-688, 2000.

26. Jones PA and Taylor SM: Cellular differentiation, cytidine analogues and DNA methylation. Cell 20: 85-93, 1980.

27. Cramer JM, Scarsdale JN, Walavalkar NM, Buchwald WA, Ginder GD and Williams DC Jr: Probing the dynamic distribution of bound states for methylcytosine-binding domains on DNA. J Biol Chem 289: 1294-1302, 2014.

28. Wood KH and Zhou Z: Emerging molecular and biological functions of MBD2, a reader of DNA methylation. Front Genet 7: 93 , 2016.

29. Quan H, Zhou F, Nie D, Chen Q, Cai X, Shan X, Zhou Z, Chen K, Huang A, Li S and Tang N: Hepatitis C virus core protein epigenetically silences SFRP1 and enhances HCC aggressiveness by inducing epithelial-mesenchymal transition. Oncogene 33: 2826-2835, 2014.

30. Desai MA, Webb HD, Sinanan LM, Scarsdale JN, Walavalkar NM, Ginder GD and Williams DC Jr: An intrinsically disordered region of methyl-CpG binding domain protein 2 (MBD2) recruits the histone deacetylase core of the NuRD complex. Nucleic Acids Res 43: 3100-3113, 2015.

31. Berger $J$ and Bird A: Role of MBD2 in gene regulation and tumorigenesis. Biochem Soc Trans 33: 1537-1540, 2005

32. Tuğrul M, Paixão T, Barton NH and Tkačik G: Dynamics of transcription factor binding site evolution. PLoS Genet 11: e1005639, 2015.

33. Liu Y, Toh H, Sasaki H, Zhang $X$ and Cheng $X$ : An atomic model of Zfp57 recognition of $\mathrm{CpG}$ methylation within a specific DNA sequence. Genes Dev 26: 2374-2379, 2012.

34. Yin Y, Morgunova E, Jolma A, Kaasinen E, Sahu B, Khund-Sayeed S, Das PK, Kivioja T, Dave K, Zhong F, et al: Impact of cytosine methylation on DNA binding specificities of human transcription factors. Science 356: eaaj2239, 2017. 